\title{
A FULLY COMPUTERIZED SCANNING TUNNELLING MICROSCOPE
}

\author{
M. Chwialkowski, Z. Klusek, P. Kobierski, W. Olejniczak, M. Slawski \\ Department of Solid State Physics, University of Eódź \\ Pomorska 149/153, 90-236 Eódź, Poland
}

\author{
AND A. WiTEK \\ Applied Thermal Physics Laboratory, Department of Physics and Astronomy \\ University of Delaware \\ Newark DE 19716, USA,
}

\begin{abstract}
A new, fully computerized and inexpensive, scanning tunnelling microscope was designed and built. Its key design feature is the application of the high sensitivity bimorph, for the coarse positioning of the bimorph is $>0.03 \mathrm{~mm}$ and can be divided up to the 12 bit number of steps. The bimorph positioning implies that this microscope is fully computer controlled, and using of bimorph instead of inch-worm implies low cost of the unit. The microscope can create images of the surface in the constant current mode and in the imaging current mode. The presented microscope works with the $z$-axis analog or digital feedback loop optionally. All parameters of the analog and digital feedback loop are computer controlled. Due to the digital $z$-axis feedback loop benefits, electron tunneling spectroscopy mode is naturally accessible. The performance of the microscope was tested in air by imaging of surface of HOPG (high oriented pyrolytic graphite).
\end{abstract}

PACS numbers: $07.80 .+x$

\section{Introduction}

A small number of the fully computer controlled scanning tunnelling microscope (STM) designs have been reported. The instrument presented here uses the piezoelectric bimorph for the coarse adjustment of tip-to-sample distance. Convenience of operation and simplicity of the bimorph positioning allows us to use this solution previously reported in [1]. The bimorph positioning implies also that our STM could be easily fully computer controlled.

Using the bimorph made of the same piezoelectric material as scanning tripod, we avoid problems connected with the thermal drift in the vertical direction. Another feature of this apparatus is compact construction. Our objective was to achieve the maximum automatization of the operation of the STM combined with 
simplicity of the mechanical part of the system. All adjustable parameters of the electronics, including time constant in the analog feedback loop, are computer controlled. The use of the CAMAC crate allows easy extension of the electronics and software features. Our STM can work in the constant current mode as well as in the imaging current mode. The piezoelectric scanner can be easily exchanged and tripod can be used as well as piezoelectric tube. Combining the different scanners and different electronics features, we can cover wide range of the observed area of surface and wide range of the regime of work. The maximum scan range of our microscope is $6000 \AA \times 6000 \AA$ generated by a piezotube or tripod. Our STM head is HV (but not UIV) compatible.

\section{STM head design}

The STM head is shown in Fig. 1. The piezoelectric scanner on which the tip is mounted is based on the tripod design (used by a number of groups) or on the piezoelectric tube developed in [2]. The scanner and bimorph subassembly are mounted on an efficient vibration isolator consisting of a number of stainless-steel



Fig. 1. Schematic drawing of the STM design: (1) coarse adjustment screw, (2) tripod or tube scanner, (3) tunnelling tip, (4) sample, (5) computer controlled bimorph, (6) rubber foam.

plates separated by rubber foam damping elements. The head is fully demountable and both scanner types may be exchanged. The sample is mounted on a bimorph front surface which provides the second step coarse tip-to-sample approach. After the first step approach the screw is blocked and not used during the operation of the STM. The tunnelling distance is achieved only with the use of computer controlled bimorph. The bimorph was driven by 12 bit digital-to-analog converter and its sensitivity was equal to $75 \AA$ /bit. Due to the high sensitivity bimorph benefits, any optical microscope monitoring of the mounting process is not needed. The preamplifier of the tunnelling current is placed as close as possible to the STM head. During collecting of the data, the head could be placed in the foam box or high vacuum chamber optionally, in order to avoid an acoustical influence. 


\section{A digital control system}

The basic functions of the control system are:

a) $x, y$ positioning control using the tripod positioning mechanism and production of a complex control signal for a tube-type piezoelement.

b) Readout of the tunnelling current or voltage of the $z$-axis stabilizing loop with a 12 bit A/D conversion. There are two possibilities of the $z$-axis approach adjustment. First, an analog feedback circuit based on a system of operational amplifiers. The time constant is digitally controlled. Second, digital control of the fine-approach system is possible due to application of a 12 bit A/D converter. This way of control is specially useful for tunnelling spectroscopy. It requires, however, the use of an efficient control system based on a 16 bit microprocessor.

c) Control for the initial-approach circuitry of the type bimorph and ceramic tube. The tube scanner requires a digital signal shaping. It is possible under software control.

d) In order to achieve good accuracy of positioning using the 12 bit converter a regulation of amplification is used for high-voltage amplifiers controlling the piezoelectric elements. The digital regulation allows for a scanning range of $40 \AA$ in the whole $\mathrm{D} / \mathrm{A}$ converter range.

The realization of the control system is presented in Fig. 2. The high complexity of the control functions together with the need of fast execution of control operations (for example digital control of the stabilizing loop) resulted in the application of a CAMAC cassette for the control system. The cassette incorporates a 16 bit controller with $1 \mathrm{MB}$ memory (Intel 8086 microprocessor and static memory) [3].

The description of the block scheme is shown in Fig. 3. The voltage signal from the preamplifier (Fig. 4) of the tunnelling current is supplied via an input amplifier to three circuits:

a) electronic commutator system,

b) analog logarithming system,

c) system of a summing amplifier connected with the integrating circuit with an electronically controlled time constant.

Depending on the position of the electronic commutator, the appropriate value is supplied on the input of a sample and hold circuit. The value resulting from 12 bit A/D conversion during readout is supplied on the CAMAC bus. The operation of writing can be directed to one of the nine 12 bit registers. Six of them, connected to D/A converters, offer digitally adjusted voltages in the range $0-10 \mathrm{~V}$. The first of the registers together with the converter controls shifts along $x$-axis, the second - along $y$-axis and the third - along the $z$-axis, provided that the digital position stabilizing loop is used for $z$-axis. The matrix module (exchangeable depending on the used scanner) produces difference voltages necessary for tube piezodrive. The module allows for simple connections with the system of high-voltage amplifiers when a tripod-type piezoelement is used. The piezoelements are controlled by the system of high-voltage amplifiers: $A 1, A 2, A 3, A 4$. These amplifiers produce voltages from 0 to $230 \mathrm{~V}$. Their nominal amplification is equal to 25 . 
CAMAC CRATE

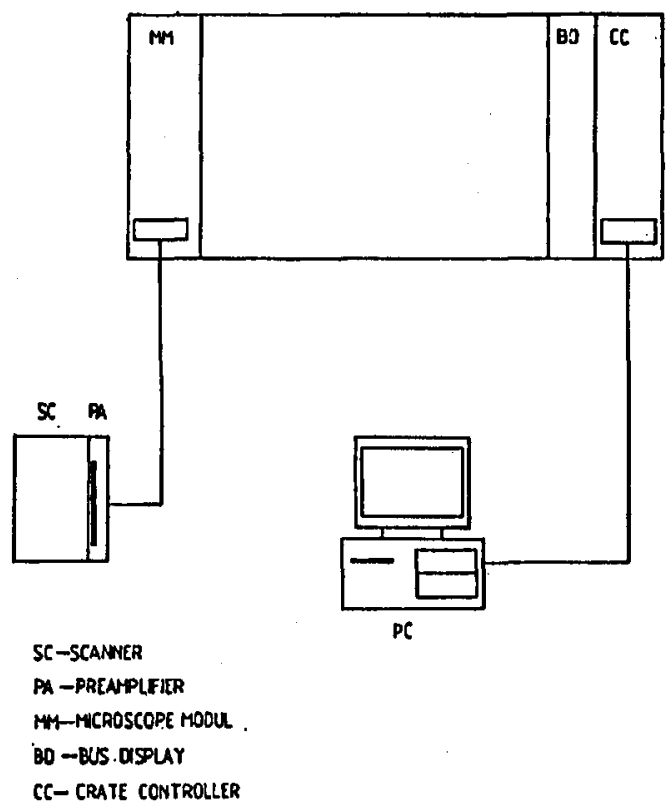

Fig. 2. Block diagram of digital control system.

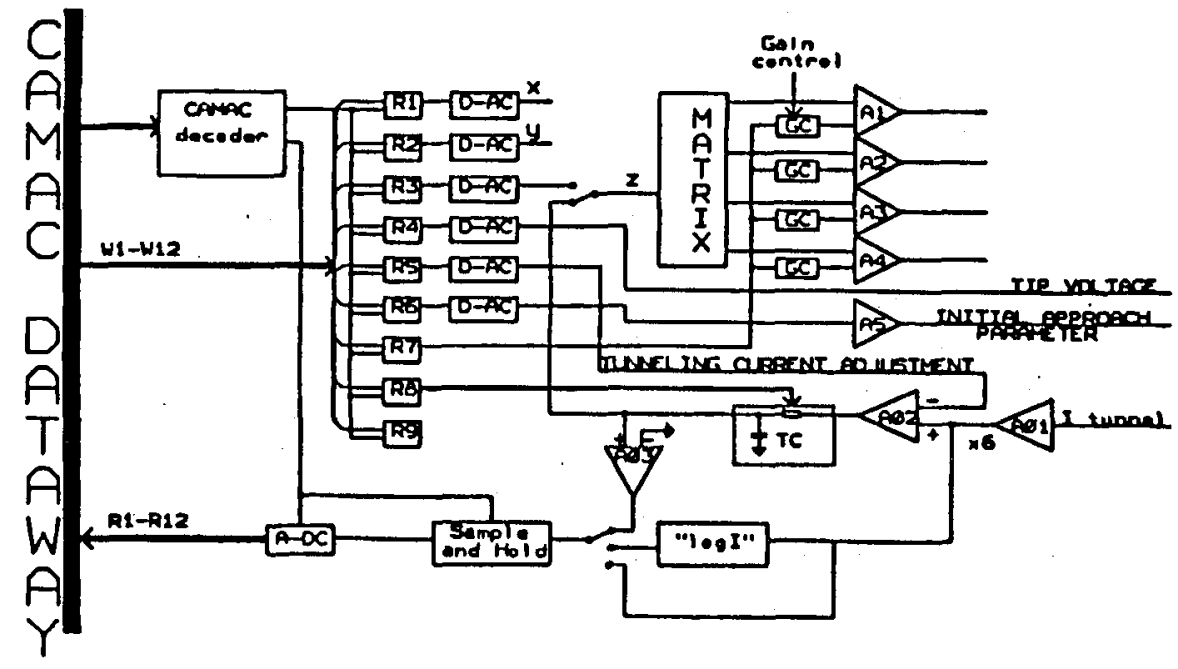

Fig. 3. Block diagram of microscope module. 


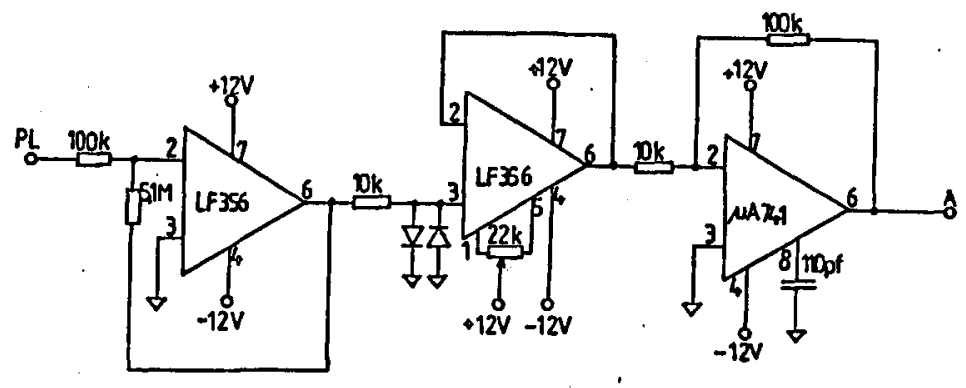

Fig. 4. Preamplifier of the tunnelling current.

The digitally controlled amplification regulation system of gain control (GC) provides an amplification reduction ratio of $1: 32$ (for precise scanning of small areas). The amplifier controlling the $z$-axis has a separate regulation of amplification.

Register $R 6$ together with a D/A converter and amplifier $A 5$ controls the system of initial approach of the sample either in bimorph or tube version. Register $R 4$ together with a D/A converter is the source which is necessary for tunnelling spectroscopy measurements. Register $R 5$ together with converter is the source of reference voltage for the summing amplifier, thereby allowing for control of the mean tunnelling current. Register $R 7$ controls the amplification regulation circuits while register $R 8$ controls the time constant of the analog regulation loop. Register $R 9$ controls the signal switching done by electronic analog switches.

\section{Software system}

There was designed and written special application software to control and manage electronic circuits. The software was written in $\mathrm{C}$ language, but some parts, were written in assembler for 8086 microprocessor. The software consists of three levels. The first level, written in assembler, is in the microcontroller system and is used to proceed basic input-output functions concerning communication between CAMAC crate and PC. The second level, written in assembler, is in microcontroller and is responsible for carrying out basic functions of STM (reading tunnelling current, changing tip position, changing time constant for analog feedback loop and other). This level of software, apart from above mentioned function, carries out digital feedback loop algorithm. Using digital feedback loop, our STM is capable of taking tunnelling spectroscopy at every pixel in the image. The third level was written in $\mathrm{C}$ language. This system supports graphic environment which enables us to service STM easily. Menu-driven screens can provide useful "templates" for user's entry of desired values for tunnelling, image control, spectroscopy, and calibration of the instrument. Graphic environment contains image processing system with some sophisticated features such as wide range of special graphic filters to correct pictures, and applies special effects including 2D fast Fourier transform (FFT). 2D real-time graphics, and 3D (after scanning) are supported, too. Full spectroscopy facilities display $I / V, \mathrm{~d} I / \mathrm{d} V$ vs. voltage curves, useful in spectroscopic analysis. STM is connected to our local area network (LAN) and this fact improves its performance especially in the area of storing large graphic files. 


\section{Conclusions}

Based on the bimorph head, the fully computerized STM was designed and built. Present-day version of STM bases on two previous models which were built by our group in $1988[4,5]$ and 1990 [6]. The following features of the innovative apparatus were achieved: computer controlled time constant in the analog

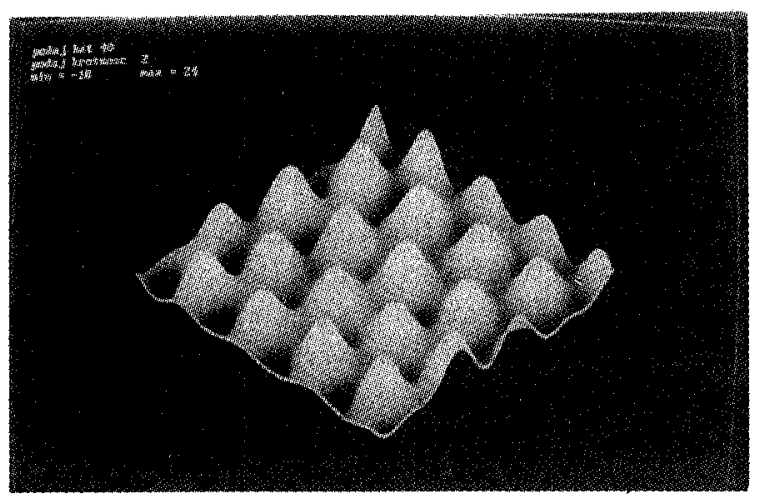

Fig. 5. High resolution image of the graphite after 2D FFT.

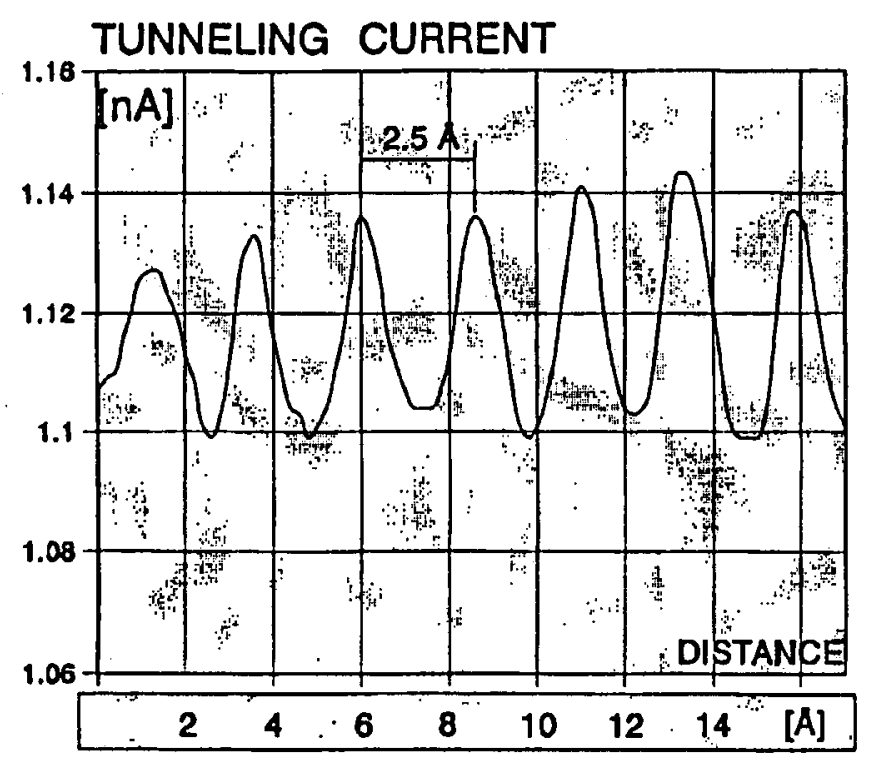

Fig. 6. The cross-section detailing the atomic separation and corrugation amplitude. The atomic separation is $2.5 \AA$. 
feedback loop, fully digitally operated feedback loop, digitally controlled tip bias. The performance of STM was tested on the HOPG. A topographic image of the IIOPG surface obtained in air is shown in Fig. 5. This figure displays the trigonal symmetry of the $\beta$ atomic sites in the surface layer of bulk graphite after 2D FFT. A $12 \AA \times 12 \AA$ atomic resolution image was taken in the imaging current mode, the sample bias was $+0.023 \mathrm{~V}$. Figure 6 presents a cross-section detailing atomic separation and the corrugation amplitude. The cross-section and adjustment of piezoclements yields an atomic separation of $2.5 \AA$, the accepted crystallographic value is $2.45 \AA$. The comparison of the most important parameters of our STM with commercially available STM is presented in Table.

TABLE

\begin{tabular}{l|l|l}
\hline \hline Specification & VG Microtech $[7]$ & Our STM \\
\hline Acquisition processor & 80286 & 8086 \\
\hline Computer system & 80386 & $8048650 \mathrm{MHz}$ \\
\hline Tunnelling current set & 12 bit DAC & 12 bit DAC \\
\hline Tunnelling voltage set & 16 bit DAC & 12 bit DAC \\
\hline$x, y$ scan & 16 bit DAC & 12 bit DAC \\
\hline$z$ axis modulation & 16 bit DAC & 12 bit DAC \\
\hline $\begin{array}{l}\text { Topography-instant current } \\
\text { and constant height modes }\end{array}$ & yes & yes \\
\hline $\begin{array}{l}\text { Cross-section and corrugation } \\
\text { amplitude }\end{array}$ & no & \\
\hline $\begin{array}{l}\text { Spectroscopy } I / V, \text { d } I / d V, \text { dual } \\
\text { polarity imaging }\end{array}$ & yes & yes \\
\hline Lateral resolution & better than $2.5 \AA$ & better than $2.5 \AA$ \\
\hline Vertical resolution & better than $0.2 \AA$ & $0.2 \AA-0.4 \AA$ \\
\hline Tip scanner & Tubular, & Tubular or Tripod, \\
& max scan range & max scan range \\
& $1 \mu$ m $\times 1 \mu \mathrm{m}$ & $6000 \AA \times 6000 \AA$ \\
\hline Vibration isolation & 4 stage viton stack & rubber spring \\
\hline Specimen drift & better than $5 \AA / \mathrm{min}$ & better than $5 \AA / \mathrm{min}$ \\
\hline sll feedback parameters under & yes & yes \\
\hline Vacuum chamber & yes & optionally \\
\hline
\end{tabular}

\section{Acknowledgments}

The autors would like to thank Prof. L. Wojtczak for helpful attention.

The paper was supported by the Project 0108: Physical Properties of the Surface and the Subsurface Layers. 


\section{References}

[1] W.J. Kaiser, R.C. Jacklevic, IBM J. Res. Dev. 30, 441 (1986).

[2] D.P.E. Smith, G. Binning, Rev. Sci. Instrum. 57, 1688 (1986).

[3] M. Nieszała, W. Olejniczak, J. Skierski, in: Proc. Conf. Surface Physics, Eódź 1989.

[4] A. Zaluska, L. Zaluski, A. Witek, Mater. Sci. Eng. A 122, 251 (1989).

[5] A. Slawska-Waniewska, A. Zaluski, A. Witek, in: Proc. 12 AJRAPT and 27 EHPRG Int. Conf. on High Pressure Science and Technology, 1989.

[6] R. Oliński, T. Zastawny, W. Olejniczak, S. Wojczuk, M. Chwiałkowski, Acta Biochim. Pol. 39, 15 (1992).

[7] STM 2000, Scanning Tunnelling Microscope for Atomic Resolution Surfaces Studies, VG MICROTECH, Bellbrook Business Park, Uckfield (UK). 